\title{
On the Nature of the Unique $\mathrm{H} \alpha$-Emitting T-Dwarf 2MASS $\mathrm{J} 12373919+6526148$
}

\author{
James Liebert \\ Steward Observatory, University of Arizona, Tucson AZ 85721, liebert@as.arizona.edu \\ and \\ Adam J. Burgasser ${ }^{1}$ \\ Massachusetts Institute of Technology, Kavli Institute for Astrophysics and Space Research, \\ 77 Massachusetts Avenue, Building 77, Cambridge MA 02139, ajb@mit.edu
}

\begin{abstract}
We explore and discount the hypothesis that the strong, continual $\mathrm{H} \alpha$ emitting T dwarf 2MASS J12373919+6526148 can be explained as a young, low gravity, very low mass brown dwarf. The source is already known to have a marginally-fainter absolute magnitude than similar $\mathrm{T}$ dwarfs with trigonometric parallax measurements, and has a tangential velocity consistent with old disk kinematics. Applying the technique of Burgasser, Burrows \& Kirkpatrick on new near infrared spectroscopy for this source, estimates of its $\mathrm{T}_{\text {eff }}$, $\log g$ and metallicity $([\mathrm{M} / \mathrm{H}])$ are obtained. $2 \mathrm{M} 1237+6526$ has a $\mathrm{T}_{\text {eff }} \approx 800-850 \mathrm{~K}$. If $[\mathrm{M} / \mathrm{H}]$ is solar, $\log g$ is as high as $\sim 5.5(\mathrm{cgs})$ and this source is older than $10 \mathrm{Gyr}$. We find a more plausible scenario to be a modestly subsolar metallicity $([\mathrm{M} / \mathrm{H}]$ $=-0.2$ ) and moderate $\log g \sim 5.0$, implying an age older than 2 Gyr and a mass greater than $0.035 \mathrm{M}_{\odot}$. The alternative explanation of the unique emission of this source, involving an interacting, close, double degenerate system, should be investigated further. Indeed, there is some evidence of a $\mathrm{T}_{\text {eff }}<500 \mathrm{~K}$ companion to $2 \mathrm{M} 1237+6526$ on the basis of a possible Spitzer IRAC [3.6]-[4.5] color excess. This excess may, however, be caused by a subsolar metallicity.
\end{abstract}

Subject headings: stars: individual ( 2MASS J12373919+6526148) — stars: low mass, brown dwarfs - techniques: spectroscopic

\footnotetext{
${ }^{1}$ Visiting Astronomer at the Infrared Telescope Facility, which is operated by the University of Hawaii under Cooperative Agreement NCC 5-538 with the National Aeronautics and Space Administration, Office of Space Science, Planetary Astronomy Program.
} 


\section{Introduction}

It is well known that the frequency and relative luminosity of $\mathrm{H} \alpha$ as a likely measure of chromospheric activity drops rapidly beyond spectral types M7 V (Kirkpatrick et al. 2000, Gizis et al. 2000, Mohanty \& Basri 2003, West et al. 2004). Very few T dwarfs have exhibited this emission at all, and generally at a very low level compared to active mid-M dwarfs, as measured by the ratio of $\mathrm{L}_{H \alpha} / \mathrm{L}_{b o l}$ (Burgasser et al. 2003). Occasionally, a vigorous flare is observed in a late $\mathrm{M}$ or $\mathrm{L}$ dwarf, causing the ratio to rocket upwards by orders of magnitude. For the most part, however, magnetic pressures built up by the likely dynamo inside these rapidly-rotating objects are not expressed at the surface, due to the inability of interior plasmas to penetrate the high resistivities of the predominantly neutral atmospheres (Mohanty \& Basri 2003).

Two exceptions to this behavior have been found, exhibiting in all observations levels of $\mathrm{H} \alpha$ emission 1-2 orders of magnitude higher in $\mathrm{L}_{H \alpha} / \mathrm{L}_{b o l}$ than any counterparts of similar spectral type. These are the M9.5 dwarf PC 0025+0047 (Schneider et al. 1991) and the T6.5 dwarf 2MASS J12373919+6526148 (hereafter 2M 1237+6526, Burgasser et al. 1999). Continual $\mathrm{H} \alpha$ activity of the former source has been observed for over a decade (Mould et al. 1994; Martín, Basri, \& Zapatero Osorio 1999). That the T dwarf seems to show similar characteristics, though over a shorter time interval so far, has suggested that emission processes in the two objects may be related.

Martín et al (1999) made the case that the strong emission lines, variable amount of optical veiling, and a claimed detection of the Li $6707 \AA$ doublet indicated that PC $0025+0447$ is a substellar object less massive than $0.06 \mathrm{M}_{\odot}$ and younger than $\sim 1$ Gyr. Their detection of weak K I and Na I resonance doublets indicated a low surface gravity. They argued, however, that the lack of an infrared excess and the fact that the $\mathrm{H} \alpha$ profile is not similar to classical $\mathrm{T}$ Tauris suggests that chromospheric activity rather than an active accretion disk is the likely source of the intense emission. If the same scenario applies to $2 \mathrm{M} 1237+6526$, this object could be a very low mass, young, very active brown dwarf. An age of 1 Gyr or less implies a maximum mass of $30 \mathrm{M}_{J}$ (Burrows et al. 2001) for an effective temperature $\mathrm{T}_{\text {eff }} \approx 800 \mathrm{~K}$ based on the Golimowski et al. (2004) scale (see their Fig. 6).

We have explored an alternative hypothesis for this source: that it is an interacting brown dwarf or double degenerate binary (Burgasser et al. 2000a, 2002a). Some possible predictions in the former paper were tested weakly in the latter paper. Monitoring of the Jband flux failed to detect significant variability at the \pm 0.025 mag level for periods of hours. Three separate measurements of the $\mathrm{H} \alpha$ line made at different times with the Keck/LRIS spectrograph between 1999 and 2001 (over a span of 1.6 yr) were inconclusive in establishing

or ruling out variability in the radial velocity. Thus, no evidence was uncovered in favor of 
the interacting binary hypothesis. On the other hand, it has not been ruled out.

In this paper, we explore the first hypothesis: that $2 \mathrm{M} 1237+6526$ is young, has low gravity, and a very active chromosphere. We begin by noting that there is already evidence against this in the literature $(\S 2)$. We present additional evidence based on infrared spectrophotometry in $\S 3$. Temperature and gravity sensitive infrared flux indices are compared with atmospheric models to estimate $\mathrm{T}_{\text {eff }}, \log g$ and the atmospheric abundances $([\mathrm{M} / \mathrm{H}])$ for the emission line object, as well as age and mass, in $\S 4$. Constraints on the properties of the hypothesized companion to $2 \mathrm{M} 1237+6526$ are discussed in $\S 5$, based in part on an apparent mid-infrared color excess detected by Spitzer observations. Results are summarized in $\S 6$.

\section{Luminosity and Kinematics}

Vrba et al. (2004) have published trigonometric parallaxes of $40 \mathrm{~L}$ and $\mathrm{T}$ dwarfs which permit the assessment of the absolute magnitude of $2 \mathrm{M} 1237+6526$ in comparison with $\mathrm{T}$ dwarfs of similar spectral type. Its absolute $J$-band magnitude of $15.88 \pm 0.13$ makes it similar to, and even marginally fainter than, other T6-T7 dwarfs with parallax measurements. The same statement applies to absolute magnitudes at $H$ and especially $K_{s}$ where it is fainter than two T7-7.5 objects. This can be seen in Figs. 2-4 of Vrba et al. It is therefore not overluminous compared with other late $\mathrm{T}$ dwarfs, as might be expected for a young brown dwarf with a larger radius (Burrows et al. 1997). This also rules out the presence of an unseen massive companion; no companion has yet been detected via high resolution imaging (Burgasser et al. 2003).

A second argument against youth is the kinematics of this source. Vrba et al. (2004) measured its tangential velocity to be $56 \pm 3 \mathrm{~km} \mathrm{~s}^{-1}$, greater than the median velocity of their sample $\left(39 \mathrm{~km} \mathrm{~s}^{-1}\right)$. In contrast, PC $0025+0047$ has a tangential velocity of only $3.6 \pm 0.4 \mathrm{~km}$

$\mathrm{s}^{-1}$ (Dahn et al. 2002). Young objects in the vicinity of the Sun typically have small relative motions, while older disk and halo objects can exhibit large velocities (eg. Wielen 1977). A full $U V W$ space motion analysis of $2 \mathrm{M} 1237+6526$ is not possible as its radial velocity has not yet been measured. However, its large tangential speed does argue against a young age.

\section{The Near Infrared Spectrum}

Far red / near infrared spectrophotometry of 2M 1237+6526 were obtained on 8 April 2006 (UT) with the SpeX spectrograph (Rayner et al. 2003) mounted on the 3-m NASA 
Infrared Telescope Facility (IRTF). Conditions were average, with light cirrus and moderate seeing $\left(1^{\prime \prime}\right.$ at $\left.J\right)$. The SpeX prism mode with a 0.5 slit was employed, providing $0.8-2.45 \mu \mathrm{m}$ spectra with an average resolution $\lambda / \Delta \lambda \approx 120$. The slit was aligned with the parallactic angle to mitigate color refraction. Six exposures of $120 \mathrm{~s}$ each were obtained at an airmass of 1.50. The A0 V star HD 99966 was observed immediately afterward for flux calibration and telluric absorption corrections. HeNeAr arc lamps and quartz lamp flat fields were also obtained for dispersion and pixel response calibration. Data were reduced using the SpeXtool package version 3.3 (Vacca, Cushing \& Rayner 2003; Cushing, Vacca \& Rayner 2004), as described in detail in Burgasser et al. (2004).

The reduced SpeX spectrum of 2M 1237+6526 is shown in Figure 1, along with those of the T6.5 dwarfs SDSS J134646.45-003150.4 (Tsvetanov et al. 2000; hereafter SD 13460031) and SDSS J175805.46+463311.9 (Knapp et al. 2004; hereafter SD 1758+4633), taken from Burgasser, Burrows \& Kirkpatrick (2006, hereafter BBK). While the threesome exhibit similar pseudo-continuum peaks typical of late $\mathrm{T}$ dwarfs at $1.27 \mu \mathrm{m}$, the emission line object shows a smaller peak at $1.57 \mu \mathrm{m}$ and a very depressed continuum peak near $2.1 \mu \mathrm{m}$ compared with the others. $\mathrm{CH}_{4}$ and $\mathrm{H}_{2} \mathrm{O}$ bands are prominent in the $H$ and $K$ bands where the last two peaks fall. However, pressure-induced $\mathrm{H}_{2}$ molecular opacity is very strong at $K$ and affects $H$ as well. Precisely because of its strong dependence on pressure, this opacity should be more sensitive than those of the other two molecules to surface gravity and metallicity. The strong dependence of the $\mathrm{K}$ band peak on $\log g$ is demonstrated in Fig. 3 of BBK. A decrease of the atmospheric metal abundance below solar metallicity also has a similar effect (cf. Saumon et al. 1994).

On the other hand, $2 \mathrm{M} 1237+6526$ has the highest $\mathrm{Y}$ band $(\sim 1.05 \mu \mathrm{m})$ peak of the three sources. The slope blueward of this peak is shaped largely by the pressure-broadened red wing of the $0.77 \mu \mathrm{m} \mathrm{K} \mathrm{I} \mathrm{doublet.} \mathrm{At} \mathrm{higher} \mathrm{photospheric} \mathrm{pressures} \mathrm{(characteristic} \mathrm{of} \mathrm{high}$ surface gravity and/or metal-deficient objects), the slope of this red wing steepens as the near-center wings deepen (Burrows \& Volobuyev 2003). Hence, the sharper $1.05 \mu \mathrm{m}$ peak of $2 \mathrm{M} 1237+6526$ is consistent with the behavior of the $K$-band peak; both result from either a high surface gravity or lower metallicity.

The $\mathrm{K} \mathrm{I}$ and $\mathrm{H}_{2}$ features thus provide evidence that $2 \mathrm{M} 1237+6526$ has the highest surface gravity and/or lowest metallicity of the three $\mathrm{T}$ dwarfs displayed in Figure 1. This also provides an explanation for why the $\mathrm{M}_{K}$ value is fainter than the comparison objects, and suggests that it has among the highest gravities of the late $\mathrm{T}$ dwarfs in the Vrba et al. parallax sample. A higher than average gravity for a brown dwarf implies a relatively high mass and older age. Subsolar metallicity can also imply a higher surface gravity. Stellar interiors deficient in heavy elements generally have smaller radii (i.e. larger gravity) at a given 
temperature, and theoretical calculations show that the same should be true for substellar entities (Burrows et al. 1993). Both scenarios imply that 2M $1237+6526$ is unlikely to be young, and may in fact be quite old. This hypothesis can be tested using the tools from BBK.

\section{Estimating $\mathbf{T}_{e f f}$ and Surface Gravity Using Spectral Indices and Constraints on the Mass and Age of $2 \mathrm{M} 1237+6526$}

BBK has demonstrated that a comparison of $\mathrm{H}_{2} \mathrm{O}$ and color ratio indices measured on the near infrared spectra of late-type $\mathrm{T}$ dwarfs to those measured on spectral models, after normalizing the latter to the well-characterized T7.5 brown dwarf companion Gliese 570D (Burgasser et al. 2000b), provides a means of disentangling the effects of $\mathrm{T}_{\text {eff }}$ and surface gravity for these sources. These parameters can then be used to infer age and mass using evolutionary models or a measured bolometric luminosity. The technique described in BBK was used to estimate ages for SD 1346-0031 and SD 1758+4633 of 0.3-0.9 and 1.0-4.9 Gyr, respectively. The spectral comparison of Fig. 1 suggests that $2 \mathrm{M} 1237+6526$ is older still.

We implemented the BBK technique for the spectrum of $2 \mathrm{M} 1237+6526$ by comparing two pairs of indices $\left(\mathrm{H}_{2} \mathrm{O}-\mathrm{J}\right.$ and $\mathrm{K} / \mathrm{H}, \mathrm{H}_{2} \mathrm{O}-\mathrm{H}$ and $\mathrm{K} / \mathrm{J}$ ) with two model sets (Burrows, Sudarsky \& Hubeny 2005; Allard et al. 2001). Figure 2 display the resulting best fit $\mathrm{T}_{\text {eff }}$ and $\log g$ phase spaces for this source, assuming solar metallicity and $10 \%$ uncertainty in the spectral indices. While there are slight differences between these comparisons, all four are consistent with high surface gravity $(\log g=5.2-5.5 \mathrm{cgs})$ and low $\mathrm{T}_{\text {eff }}(740-860 \mathrm{~K})$. The fit using the Burrows et al. models and $\mathrm{H}_{2} \mathrm{O}-\mathrm{J}$ and $\mathrm{K} / \mathrm{H}$ indices (the nominal set for $\mathrm{BBK}$ ) is quite similar to that for 2MASS J00345157+0523050 (Burgasser et al. 2004), another T6.5 that exhibits strong $K$-band suppression and a large proper motion, for which BBK estimate an age of 3.4-6.9 Gyr.

As discussed above, subsolar metallicity can also lead to enhanced $\mathrm{H}_{2}$ absorption. Hence, it is necessary to assess whether the spectrum of $2 \mathrm{M} 1237+6526$ could match that of a metalpoor brown dwarf. We repeated the parameter fit analysis using the $\mathrm{H}_{2} \mathrm{O}-\mathrm{J}$ and $\mathrm{K} / \mathrm{H}$ indices and subsolar metallicity models from Burrows et al. spanning $[\mathrm{M} / \mathrm{H}]=-0.5$ to 0 . Results are shown in Figure 3. As $[\mathrm{M} / \mathrm{H}]$ is lowered, the best fit $\mathrm{T}_{\text {eff }}$ stays around $750-850 \mathrm{~K}$, while the best fit $\log g$ generally decreases. At $[\mathrm{M} / \mathrm{H}]=-0.5$, the best fit $\log g$ has dropped a full dex from the solar abundance fit to 4.5 .

There is a clear degeneracy between $\log g$ and $[\mathrm{M} / \mathrm{H}]$ parameters based on this twoindex fitting procedure. Ideally, one would like to use a third index, specifically sensitive 
to metallicity or gravity, to break the degeneracy. However, the best candidate for such an index, the $\mathrm{Y} / \mathrm{J}$ ratio (ratio between the $\mathrm{Y}$ - and J-band pseudocontinuum flux peaks), is highly sensitive to the $\mathrm{K} \mathrm{I}$ line broadening physics, for which more recent calculations (Burrows \& Volobuyev 2003) are not included in the Burrows et al. subsolar metallicity models (BBK). As an alternative, we compared absolute spectral fluxes for 2M 1237+6526 to those of the Burrows et al. models, since a more metal-poor (less opacity) and lower surface gravity (larger radius) object is overall more luminous. Figure 4 compares the absolute flux calibrated spectrum for $2 \mathrm{M} 1237+6526$ to the best fitting $[\mathrm{M} / \mathrm{H}]=0$ and -0.5 models from the index analysis. The data lie between these two extremes and are inconsistent with them at the $1 \sigma$ flux level, suggesting that $2 \mathrm{M} 1237+6526$ is most likely to be slightly metalpoor $([\mathrm{M} / \mathrm{H}] \sim-0.2)$ brown dwarf with a moderate surface gravity $(\log g \sim 5.0)$. There are naturally several caveats to consider with this interpretation, including uncertainties in the $\mathrm{T}_{\text {eff }}$ and $\log g$ determinations, systematic errors in the models and the possibility of excess light from a faint companion. Nevertheless, the fact that this object does not exhibit the more pronounced metallicity features of the peculiar T6 2MASS J09373487+2931409 (Burgasser et al. 2002b; hereafter 2M 0937+2931), for which BBK derive $[\mathrm{M} / \mathrm{H}]=-0.1$ to -0.4 , argues for a metallicity closer to solar.

The $\mathrm{T}_{\text {eff }}$ and $\log g$ derived for $2 \mathrm{M} 1237+6526$ can be used to infer the mass and age for this source using the Burrows et al. (1997) evolutionary models. For solar metallicity, the four index analyses in Fig. 1 combined yield an age of 5-10 Gyr and $\mathrm{M}=0.043-0.065 \mathrm{M}_{\odot}$. This is both old and relatively massive for a brown dwarf. The lowest metallicity fit $([\mathrm{M} / \mathrm{H}]$ $=-0.5)$ yields a significantly younger age $(\sim 0.4 \mathrm{Gyr})$ and lower mass $\left(\sim 0.015 \mathrm{M}_{\odot}\right)$, but for the reasons discussed above this interpretation is not favored. Rather, for $\log g \gtrsim 5.0$ and

the best fit $\mathrm{T}_{\text {eff }} \mathrm{S}(800-850 \mathrm{~K}), 2 \mathrm{M} 1237+6526$ is likely to be older than 2 Gyr and more massive than $0.035 \mathrm{M}_{\odot}$. This conclusion is supported by the deduced mass of this object from the estimated $\mathrm{T}_{\text {eff }}$ and $\log g$ values and the measured bolometric luminosity (Vrba et al. (2004), which yield $\mathrm{M}=L g / 4 \pi G \sigma T_{\text {eff }}^{4} \approx 0.04 \mathrm{M}_{\odot}$, albeit with significant uncertainty.

\section{Constraints on the Hypothetical Companion of 2M 1237+6526: Has It Been Detected?}

If $2 \mathrm{M} 1237+6526$ has a companion overfilling its Roche lobe, we concluded in Burgasser et al. (2000) that this companion's mass must be $<63 \%$ that of the primary. Hence, for a primary with mass $0.04 \mathrm{M}_{\odot}\left(0.065 \mathrm{M}_{\odot}\right)$ at an age of $2 \mathrm{Gyr}(10 \mathrm{Gyr})$, then the companion must have a mass $<0.025 \mathrm{M}_{\odot}\left(<0.04 \mathrm{M}_{\odot}\right)$ and $\mathrm{T}_{\text {eff }}<650 \mathrm{~K}(<550 \mathrm{~K})$. The hypothesized semi-detached companion, under these conditions, must be substantially cooler than its 
primary, and also cooler than any currently-known $\mathrm{T}$ dwarf.

Could such a companion have been detected in Spitzer -IRAC 3.6-8 $\mu \mathrm{m}$ observations of $2 \mathrm{M} 1237+6526$ ? We show in Figure 5 a color magnitude diagram for the two most sensitive IRAC bands, the absolute $3.6 \mu \mathrm{m}$ magnitude vs. the [3.6]-[4.5] color for objects $\mathrm{T} 5$ and later with trigonometric parallaxes, including two known mid-T binaries with components of similar brightness. The data are taken from Patten et al. (2006), plus values for the T7.5 companion to HD 3651 are taken from Luhman et al. (2006). Also shown are synthetic photometry based on the models similar to those of Burrows, Sudarsky \& Lunine (2003). As discussed in Patten et al. (2006), the disagreement between the models and data in this plot likely arises from overestimated $4.5 \mu \mathrm{m}$ fluxes in the models. This is likely due to underestimated abundances of CO, that appears to be enhanced by vertical upwelling (Noll, Geballe, \& Marley 1997; Oppenheimer et al. 1998; Saumon et al. 2000). Absorption due to this molecule's fundamental band at $\sim 4.67 \mu \mathrm{m}$ probably causes the observed fluxes to be weaker than the models predict, and therefore the [3.6]-[4.5] colors to be bluer.

Trends in the data suggest a blue envelope of [3.6]-[4.5] colors that parallels similar trends in the models. This envelope, which we approximate as

$$
[3.6]-[4.5] \geq-7.05+0.58 M_{[3.6]}
$$

over the range $14.0<M_{[3.6]}<15.3$, should trace out the oldest and most massive single brown dwarfs, as the models predict that lower surface gravities (i.e., younger ages) yield redder colors. Most of the T5-T8 dwarfs from this sample lie close to the envelope (with small color dispersion, although there is some spread in $M_{[3.6]}$ along the envelope amongst objects in a given spectral subclass, possibly due to classification uncertainties). 2M 1237+6526, on the other hand, sits modestly above or redward of this envelope. As it is an apparently old source, this excess suggests the presence of a faint, unresolved companion.

By constraining the primary to lie on the the envelope line $\left(M_{[3.6]} \approx 14.4,[3.6]-[4.5]\right.$ $\approx 1.3$ ) - marked by a green circle - we show the effect of adding a companion with $\mathrm{T}_{\text {eff }}=$ $500 \mathrm{~K}, \log \mathrm{g}=5.0$ and solar composition, based on theoretical photometry calculated for us by A. Burrows. This companion has $M_{[3.6]}=17.28$, [3.6]-[4.5] $=3.12$. Coadding the fluxes in each of the two bands places the combined-light binary at the position of the red circle $\left(M_{[3.6]}=14.33,[3.6]-[4.5]=1.58\right)$. Thus, if our speculation is correct that $2 \mathrm{M} 1237+6526$ has a cooler companion, it is probably more than $300 \mathrm{~K}$ cooler than its primary, which is consistent with the constraints placed on the companion by the interacting binary scenario.

Note also that both the T6p 2M 0937+2931 and the T6.5 2MASS J10475385+2124234 (Burgasser et al. 1999; hereafter 2M 1047+2124; not plotted in the figure as it has values nearly identical to $2 \mathrm{M} 1237+6526)$ also lie redward of the proposed envelope. While it is 
possible that the latter source is young and has a low surface gravity, the former source has been shown to be an old, high surface gravity brown dwarf with subsolar metallicity (BBK). Since 2M 1237+6526 also appears to be slightly metal-deficient, its red [3.6]-[4.5] color may instead be a metallicity effect. Lower heavy element abundances would increase the atmospheric pressures, which might enhance the formation of $\mathrm{CH}_{4}$ while weakening CO. The latter could enhance the $4.5 \mu \mathrm{m}$ flux, while the former could weaken the $3.6 \mu \mathrm{m}$ flux. Thus, the IRAC colors of $2 \mathrm{M} 1237+6526$ cannot unambiguously confirm the presence of a low-mass unresolved companion.

\section{Summary and Implications}

On the basis of our spectral analysis, as well as the object's luminosity and kinematics, we conclude that $2 \mathrm{M} 1237+6526$ is likely to be a rather old, massive and slightly metal-poor brown dwarf. Youthful activity, which is cited as the source for the emission properties of PC 0025+0047, cannot explain this T dwarf's persistent $\mathrm{H} \alpha$ emission.

Other possibilities, including accretion from a close companion, are worth further investigation. The "tests" of the double degenerate hypothesis involving a low rate of transfer have not been conclusive. The problem with searching for possible radial velocity variations was described in the Introduction. The absence of large infrared variations sought in the Burgasser et al. (2002a) study rules out only a limited phase space for the interacting binary model. The IRAC photometry suggests a [3.6]-[4.5] color excess which could result from the presence of an unresolved cool companion, but the excess may also be attributable to a lower metallicity. Any companion would have to be appreciably lower in mass, and probably at least $300 \mathrm{~K}$ cooler. Generally speaking, as model atmospheres are improved to predict more accurate $4.5 \mu \mathrm{m}$ fluxes, including the dependence on metallicity, searching for an excess in this band may result in the detection of unresolved companions later in type than any currently-known T dwarf.

We thank our telescope operator Eric Volquardsen and instrument specialist John Rayner, for their support during the SpeX observations. Helpful discussions with Michael Cushing are acknowledged, and we thank him for help with the IRAC magnitude system. We also thank Adam Burrows for providing additional theoretical Spitzer IRAC photometry for our analysis. Our referee, Gibor Basri, provided a useful critique of our original manuscript that greatly improved it. This publication makes use of data products from the Two Micron All Sky Survey, a joint project of the University of Massachusetts and the Infrared Processing and Analysis Center/California Institute of Technology, funded by the National Aerospeace 
and Space Administration and the National Science Foundation. The authors wish to extend special thanks to those of Hawaiian ancestry on whose sacred mountain we are privileged to be guests.

\section{REFERENCES}

Allard, F., Hauschildt, P. H., Alexander, D. R., Tamanai, A., \& Schweitzer, A. 2001, ApJ, 556,357

Burgasser, A. J., Burrows, A., \& Kirkpatrick, J. D. 2006, ApJ, 639, 1095 (BBK)

Burgasser, A. J., Kirkpatrick, J. D., Liebert, J., \& Burrows, A. 2003, ApJ, 594, 510

Burgasser, A. J., Kirkpatrick, J. D., Reid, I. N., Brown, M. E., Miskey, C. L., \& Gizis, J. E. 2003, ApJ, 586, 512

Burgasser, A. J., Kirkpatrick, J. D., Reid, I. N., Liebert, J., Gizis, J. E., \& Brown, M. E. 2000a, AJ, 120, 473

Burgasser, A.J., Liebert, J., Kirkpatrick, J.D., \& Gizis, J.E. 2002a, AJ, 123, 2744.

Burgasser, A.J., McElwain, M.W., Kirkpatrick, J.D., Cruz, K.L., Tinney, C.G., \& Reid, I.N. 2004, AJ, 127, 2856

Burgasser, A. J., et al. 1999, ApJ, 522, L65

Burgasser, A. J. et al. 2000b, ApJ, 531, L57

Burgasser, A. J., et al. 2002b, ApJ, 564, 421

Burrows, A., Hubbard, W.B., Lunine, J.I., \& Liebert, J. 2001, Rev. Mod. Phys., 73, 719

Burrows, A., Hubbard, W.B, Saumon, D., \& Lunine, J.I. 1993, ApJ, 406, 158

Burrows, A., Sudarsky, D., \& Lunine, J. I. 2003, ApJ, 596, 587

Burrows, A., \& Volobuyev, M. 2003, ApJ, 583, 985

Burrows, A., et al. 1997, ApJ, 491, 856

Cushing, M.C., Vacca, W.D., \& Rayner, J.T. 2004, PASP, 116, 362

Dahn, C. C., et al. 2002, AJ, 124, 1170

Golimowski, D., et al. 2004, AJ, 127, 3516

Gizis, J.E., Monet, D.G., Reid, I.N., Kirkpatrick, J.D., Liebert, J. \& Williams, R.J. 2000, AJ, 120, 1085

Kirkpatrick, J.D., Reid, I.N., Liebert, J., Gizis, J.E., Burgasser, A.J., Monet, D.G., Dahn, C.C., Nelson, B., \& Williams, R.J. 2000, AJ, 120, 447 
Knapp, G. R., et al. 2004, AJ, 127, 3553.

Luhman, K.L. et al. 2006, ApJ, in press

Martín, E.L., Basri, G., \& Zapatero Osorio, M.R. 1999, AJ, 118, 1005

Mohanty, S. \& Basri, G. 2003, ApJ, 583, 451

Mould, J., Cohen, J., Oke, J.B., \& Reid, I.N. 1994, AJ, 107, 222

Noll, K. S., Geballe, T. R., \& Marley, M. S. 1997, ApJ, 489, L87

Oppenheimer, B. R., Kulkarni, S. R., Matthews, K., van Kerkwijk, M. H. 1998, ApJ, 502, 932

Patten, B.M. et al. 2006, ApJ, in press (astro-ph/0607537)

Rayner, J.T., Toomey, D.W., Onaka, P.M., Denault, A.J., Stahlberger, W.E., Vacc, W.D., Cushing, M.C., \& Wang, S. 2003, PASP, 115, 362

Saumon, D., Bergeron, P., Lunine, J. I., Hubbard, W. B., \& Burrows, A. 1994, ApJ, 424, 333

Saumon, D., Geballe, T.R., Leggett, S.K., Marley, M.S., Freedman, R.S., Lodders, K., Fegley, B., Jr., \& Sengupta, S.K. 2000, ApJ, 541, 374

Schneider, D.P., Greenstein, J.L., Schmidt, M. \& Gunn, J.E. 1991, AJ, 102, 1180

Tsvetanov, Z. I., et al. 2000, ApJ, 531, L61

Vacca, W.D., Cushing, M.C., \& Rayner, J.T. 2003, PASP, 115, 389

Vrba, F.J. et al. 2004, AJ, 127, 2948

West, A.A. et al. 2004, AJ, 128, 426

Wielen, R. 1977, A\&A, 60, 263 


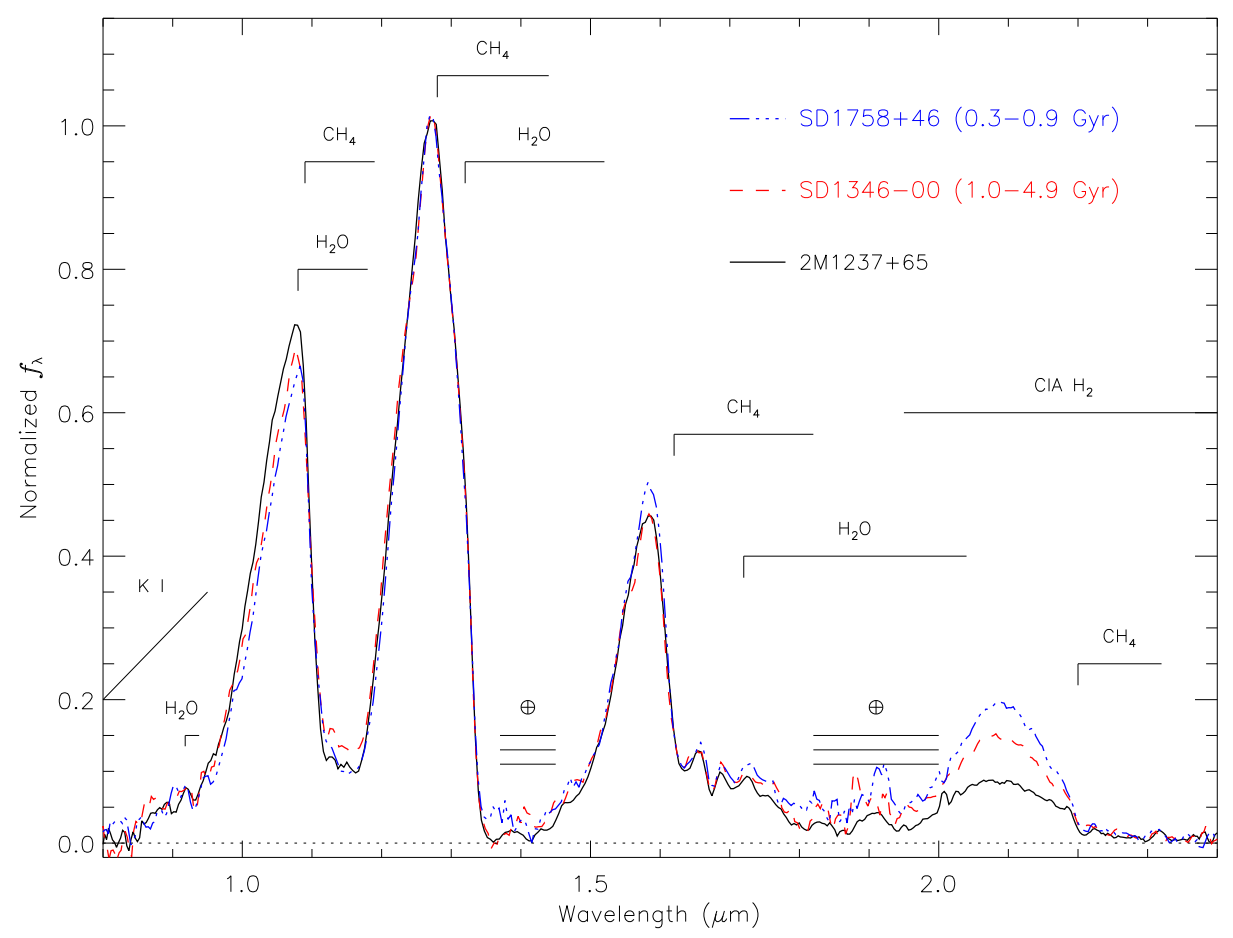

Fig. 1. - SpeX spectra of the T6.5 dwarfs 2M 1237+6526 (black solid line), SD 1346-0031 (red dashed line) and SD 1758+4633 (blue dot dashed line). All data are normalized at 1.27 $\mu \mathrm{m}$. Major spectral features are labelled, as well as telluric absorption bands ( $\oplus$ symbols). The spectra are largely identical, with the exception of significant differences in the peak $K$-band flux and slight differences in the $1.05 \mu \mathrm{m} Y$-band and $1.6 \mu \mathrm{m} H$-band peaks. Age estimates from BBK for SD 1346-0031 and SD 1758+4633 are listed. 

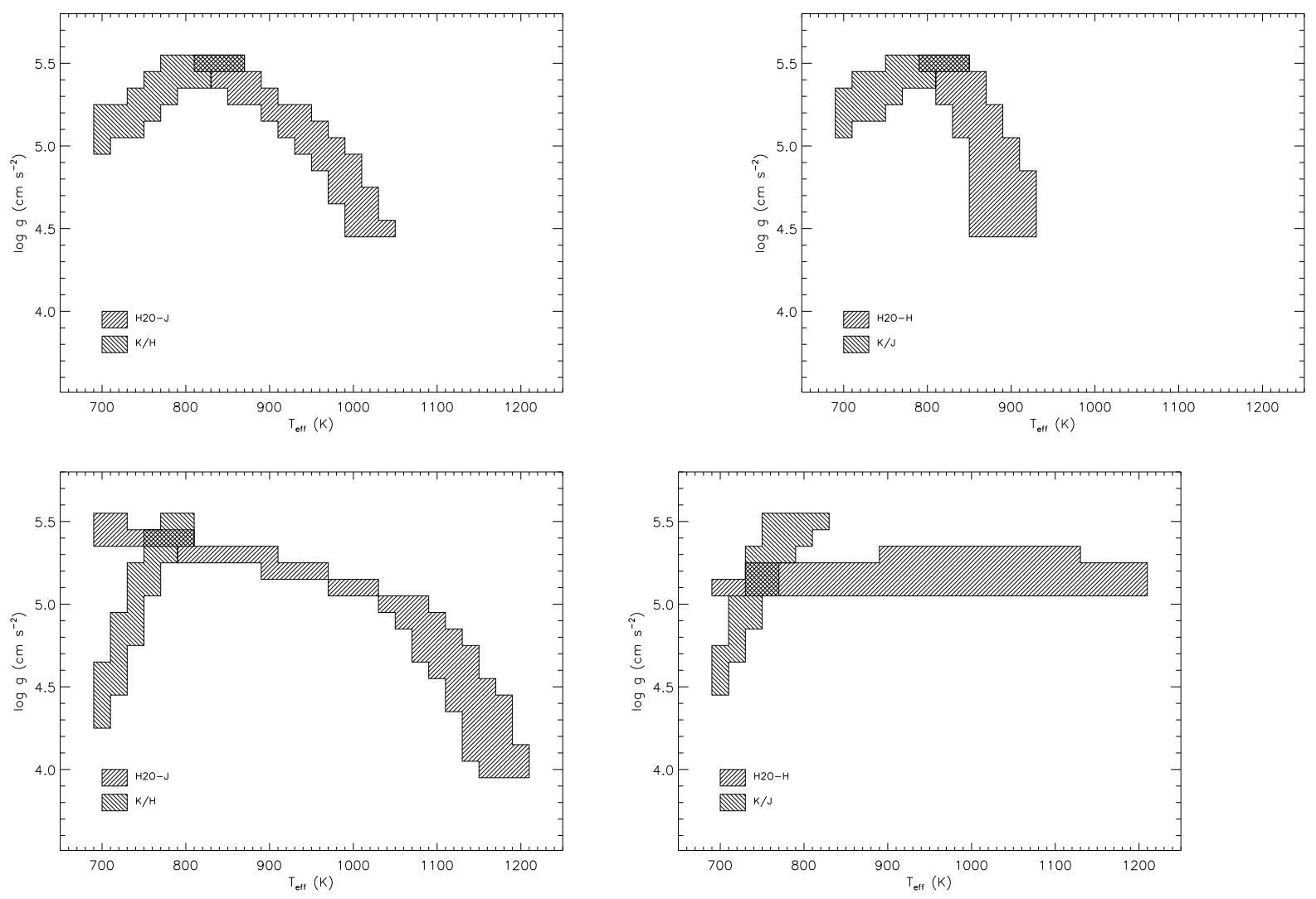

Fig. 2.- $\mathrm{T}_{\text {eff }}$ and gravity constraints for $2 \mathrm{M} 1237+6526$ based on the method of BBK. The top panels show index comparisons to the models of Burrows, Sudarsky \& Hubeny (2005), the bottom panels show comparisons to the COND models of Allard et al. (2001). The left panels compare $\mathrm{H}_{2} \mathrm{O}-\mathrm{J}$ and $\mathrm{K} / \mathrm{H}$ indices, while the right panels compare $\mathrm{H}_{2} \mathrm{O}-\mathrm{H}$ and $\mathrm{K} / \mathrm{J}$ indices. All four comparisons yield similar results, although the $\mathrm{H}_{2} \mathrm{O}-\mathrm{H}$ and $\mathrm{K} / \mathrm{J}$ comparison to the COND models yield somewhat lower $\mathrm{T}_{\text {eff }} \mathrm{s}$ and gravities. 

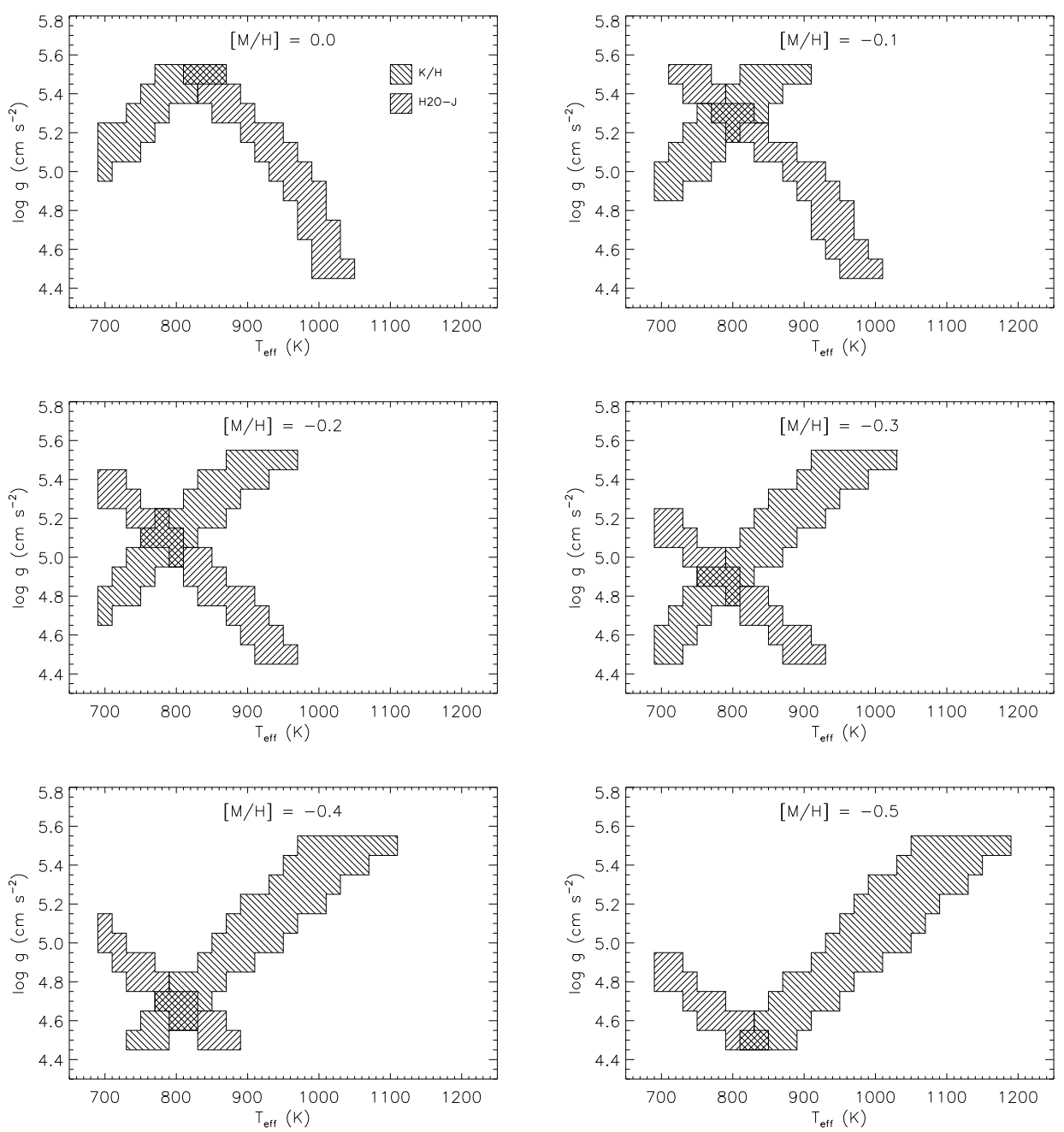

Fig. 3.- Same as Figure 2, but comparing $\mathrm{H}_{2} \mathrm{O}-\mathrm{J}$ and $\mathrm{K} / \mathrm{H}$ indices for subsolar metallicity models from Burrows, Sudarsky \& Hubeny (2005) spanning $[\mathrm{M} / \mathrm{H}]=0$ to -0.5 . Note that lower metallicity models permit lower surface gravities, and hence lower mass and younger age constraints for this pair of indices. 


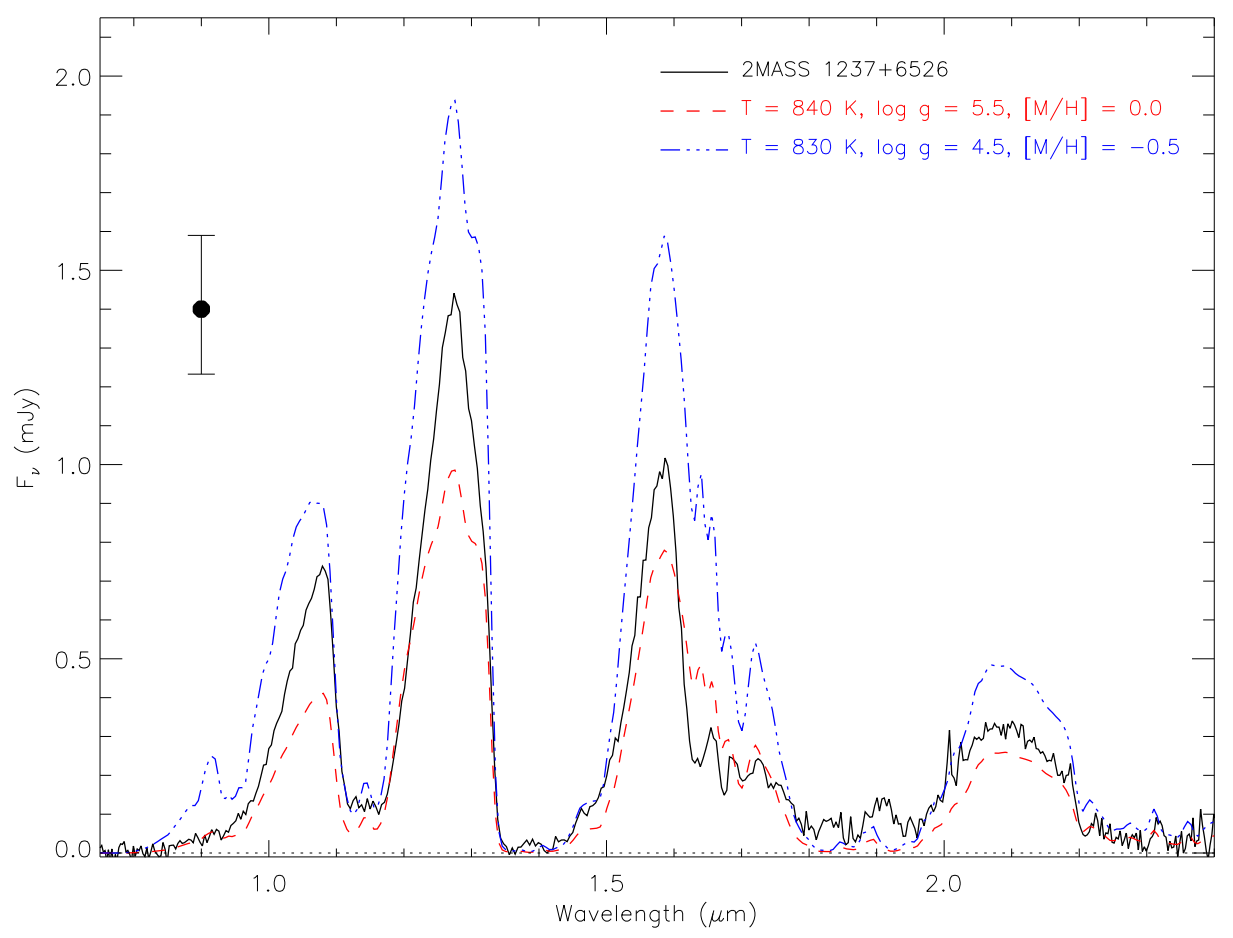

Fig. 4. - Comparison of absolute spectral fluxes $\left(F_{\nu}\right)$ for $2 \mathrm{M} 1237+6526$ (black solid line) to theoretical models for the best fitting $[\mathrm{M} / \mathrm{H}]=0$ (red dashed line; $\mathrm{T}_{\text {eff }}=840 \mathrm{~K}$ and $\log g=5.5$ ) and $[\mathrm{M} / \mathrm{H}]=-0.5$ (blue dot-dashed line; $\mathrm{T}_{\text {eff }}=830 \mathrm{~K}, \log g=4.5$ ) parameters. Uncertainty in the absolute calibration of $2 \mathrm{M} 1237+6526$, based on $J$-band photometry and parallax measurements from Vrba et al. (2004), is indicated by the error bar in the upper left corner of the plot. The spectral data appear to favor an intermediate metallicity $([\mathrm{M} / \mathrm{H}]$ $\sim-0.2)$ and surface gravity ( $\log g \gtrsim 5.0$ ) for this source, assuming no significant contribution from an unseen companion. 


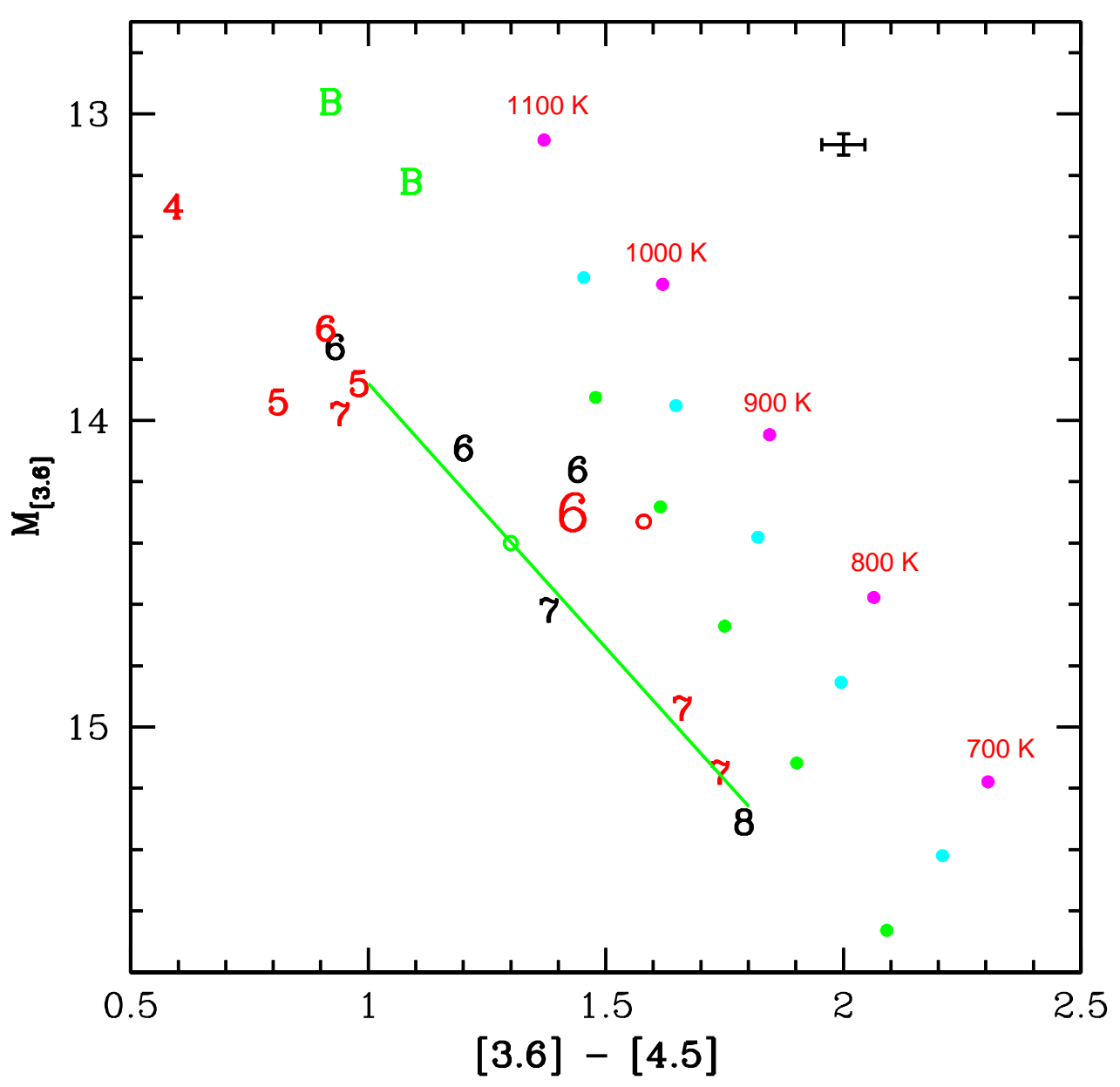

Fig. 5.- The $\mathrm{M}_{[3.6]}$ vs. [3.6]-[4.5] diagram for $\mathrm{T}$ dwarfs later than T4.0 with trigonometric parallaxes, observed in Patten et al. (2006) and Luhman et al. (2006). Each spectral class is plotted as a black numeral if the subtype is exact, or as a red color if there is 0.5 subtype to be added. 2M 1237+6526 is shown as the enlarged red "6" (for T6.5). To avoid confused symbols, 2M $1047+2124$ with virtually identical values to $2 \mathrm{M} 1237+6526$ is not plotted. Photometry for two known, equal-brightness T dwarf binaries (2M15344984-2952274 and 2M12255432-2739466, Burgasser et al. 2003) are indicated by the "B" symbols. Filled circles are synthetic photometry based on the models of Burrows, Sudarsky \& Lunine (2003) as reported in Patten et al. (2006) for $\mathrm{T}_{\text {eff }}$ as labelled, for $\log g$ values of (from right to left in cgs units) 4.5 (magenta), 5.0 (cyan), and 5.5 (green). The solid line delineates our proposed "envelope" of [3.6]-[4.5] colors for $14.0<M_{[3.6]}<15.3$, as suggested by the data (Equation 1). According to the models, this envelope should correspond to the oldest, single brown dwarfs in the sample. The green and red open circles indicate the estimated position of a high gravity $\mathrm{T} 6.5$ and show the effect of adding a $\mathrm{T}_{\text {eff }}=500 \mathrm{~K}$ companion to that source, as discussed in the text. 